\title{
Congenital dyserythropoietic anemia and drug-induced liver injury present as bland cholestasis: A case report
}

\author{
YUE HAN $^{1,2^{*}}$, YAN ZHUANG $^{1 *}$, WEILIANG TANG ${ }^{1 *}$, LU CHEN $^{1 *}$, \\ YEJING CHEN $^{3}$, QIMING GONG ${ }^{1}$ and XINXIN ZHANG ${ }^{1,2,4}$
}

\author{
${ }^{1}$ Department of Infectious Diseases, Research Laboratory of Clinical Virology, Ruijin Hospital, Shanghai Jiao Tong University \\ School of Medicine; ${ }^{2}$ Sino-French Research Center for Life Sciences and Genomics, Ruijin Hospital, Shanghai 200025; \\ ${ }^{3}$ Department of Infectious Diseases, Qionghai People's Hospital, Hainan 571400; ${ }^{4}$ Clinical Research Center, \\ Ruijin Hospital North Campus, Shanghai Jiao Tong University School of Medicine, Shanghai 200025, P.R. China
}

Received November 2, 2020; Accepted February 2, 2021

DOI: $10.3892 /$ etm.2021.9887

\begin{abstract}
Anemias and drug-induced liver injury(DILI) are separate disorders, which are difficult to diagnose. The clinical effects of DILI vary among individuals. However, the outcome determinants remain to be fully established. To the best of our knowledge, the role of anemia in DILI has yet to be reported. The present study reported on the case of one Chinese patient (male; age, 21 years) who experienced obvious drug-induced cholestasis. Of note, the hepatocyte injury was minimal compared with that in previously reported cases treated with the same drug. In addition, the patient suffered from mild hemolytic anemia with no obvious cause. A genetic pedigree analysis revealed compound heterozygous mutations in the congenital anemia-associated gene codanin 1, including the novel rare p.R1067H mutation. Treatment with ursodeoxycholic acid alone sufficed and the outcome was good. Therefore, whilst chronic hemolysis predisposed the liver to cholestasis, it could have shielded the liver from further injuries, since bilirubin, a by-product of hemolysis, is a known antioxidant. The results of the present study indicated that genetic screening may be used for the diagnosis of liver injury concurring with undiagnosed anemia.
\end{abstract}

Correspondence to: Professor Qiming Gong or Professor Xinxin Zhang, Department of Infectious Diseases, Research Laboratory of Clinical Virology, Ruijin Hospital, Shanghai Jiao Tong University School of Medicine, Building 36, 197 Ruijin Er Road, Shanghai 200025, P.R. China

E-mail: gongqm@hotmail.com

E-mail: zhangx@shsmu.edu.cn

*Contributed equally

Key words: rare congenital anemia, cholestasis, hyperbilirubinemia, ephedra-induced, protective

\section{Introduction}

Hereditary anemias are a group of common but highly pleiotropic disorders, exhibiting great molecular disparity. As highly heterogenous hematological disorders (1), the clinical knowledge of hereditary anemias remains poor. To date, $\geq 70$ genes have been identified to be involved in anemia (2). Mutations in these genes occur with low to rare frequency. The mechanisms underlying the onset of anemias include deficiencies in regeneration capacity, red blood cell (RBC) deformities, enzymatic deficiencies and other aspects of hematopoiesis. Precise diagnosis is of paramount importance, since the therapeutic approaches for anemias and contraindications are distinctly different (3). Furthermore, verifying genetic defects in adults is always challenging, largely due to the low penetrance rate, pleiotropic expression and multifactorial mechanisms. Therefore, the diagnosis and management of anemia remain challenging.

Drug-induced liver injury (DILI) is a potentially serious adverse reaction $(4,5)$. Current diagnostic scales heavily rely on causality assessment, such as the Roussel Uclaf Causal Relationship Assessment Method (RUCAM) scoring system (6), partially due to the lack of knowledge on its determinants. Downstream intrahepatic cellular events such as oxidative stress may aggravate liver injury following the initial insult $(7,8)$. The phenotype of DILI may mimic almost any other liver disorder. The clinical presentation may vary significantly among treated patients, even following treatment with the same substance. Therefore, determining protective and predisposing factors remains difficult. The overlap of DILI with a rare form of hereditary anemia has not been previously reported. The present study reported on a case of DILI presenting with bland cholestasis in the context of a chronic rare anemia. The potential interactions between these two disorders were evaluated and discussed.

\section{Materials and methods}

Clinical presentation. An otherwise healthy 21-year-old male was initially admitted to Ruijin Hospital (Shanghai, China) in March 2020 due to afebrile painless jaundice for 20 days. The 
patient was in a normal state until he underwent laparoscopic cholecystectomy after several episodes of abdominal pain and cholelithiasis in September 2019. No transfusion was required during the surgery. In October 2019, the patient developed generalized pruritus of unclear etiology. The patient underwent self-treatment with ephedra (9), which slightly improved the symptoms. In the meantime, the patient also complained of fatigue and anorexia. The patient noticed yellowish eyes and dark urine, which prompted a clinical evaluation. In addition, the patient reported pale stool, which was not observed during hospitalization. The paternal grandfather of the patient had died of cirrhosis at 36 years of age due to unknown etiology. Apart from the above medical and surgical history, the patient's medical history was unremarkable. The patient had no allergies and did not smoke or drink alcohol. On arrival, the patient's vital signs were stable and normal and he was conscious. On examination, the patient had icterus with jaundiced skin, with no visible lesions. The abdomen was distended and the spleen and liver were non-palpable below the costal margin. The remainder of the physical examination was unremarkable. Written informed consent for data usage in scientific research, including genetic testing and publication of the results was provided by the patient and his parents.

Next-generation sequencing and Sanger confirmation. Given the family history of cirrhosis, whole-exome sequencing of the pedigree, including the proband and his parents was performed to detect any possible genetic abnormalities. Genomic DNA was extracted using QIAamp DNA Blood Mini Kit (Qiagen, Inc.) from peripheral blood and was randomly fragmented using Covaris S220 Focused-ultrasonicator system (Covaris Inc.) to an average size of 180-280 bp. Libraries were constructed using paired-end protocols from Illumina, Inc. The fragmented DNA was captured using the Agilent SureSelect Human All ExonV6 kit (Agilent Technologies Inc.) and sequencing was performed on the Illumina Novaseq 6000 platform (Illumina Inc.). Paired-end $150 \mathrm{bp}$-long reads with a coverage of $\geq 10 \mathrm{X}$ for $99 \%$ of the genome (mean coverage, x100) were generated. Candidate variants, amplified by PCR, were confirmed by Sanger sequencing according to Applied Biosystems ${ }^{\circledR}$ 3730/3730xl DNA Analyzers User Guide on the ABI 3730 Prism DNA sequencer (Applied Biosystems; Thermo Fisher Scientific, Inc.) using two sets of self-designed primer pair (first pair: 5'-GGTGATATCTCCCCCTGCTC-3' and 5'-GCT TACTGCTGCGAGAACTG-3'; second pair: 5'-TTATCCTGC CCAAGTCCCAG-3' and 5'-ATTTGGGGGATCAGGCAA GA-3') encompassing the region of interest.

Filtering of rare variants and pedigree analysis. Rare exonic variants with a minor allele frequency of $<0.01$ were selected from the 1000 Genomes Project (10), the Exome Sequencing Project (ESP; esp6500siv2, https://esp.gs.washington. edu/drupal/) and The Genome Aggregation Database (gnomAD release v3.1, https://gnomad.broadinstitute.org/). Known pathogenic dominant or recessive variations, homozygous or compound heterozygous, as well as de novo variants, were all considered as candidate variations in the pedigree analysis.

Liver biopsy. Ultrasound-guided liver biopsy was performed and the specimen was stained with hematoxylin and eosin
(H\&E) and Masson trichrome (11) using standard protocols. Liver biopsy sample was fixed in $10 \%$ neutral phosphate buffered formalin at room temperature for $2 \mathrm{~h}$. Xylene and ethanol gradient method was applied in the dehydration and rehydration process. Paraffin was used as embedding reagent and the thickness of slices was 3-5 $\mu \mathrm{m}$. The cells were stained sequentially with hematoxylin and eosin at room temperature for 4 and $10 \mathrm{~min}$, respectively. The slides were observed under x100 and x400 magnification using light microscopy. Slides were evaluated by one experienced pathologist who was blinded to the clinicopathological data of the patient.

Treatment and follow-ups. The patient was treated with ursodeoxycholic acid (UDCA) $250 \mathrm{mg}$ t.i.d.po, starting on day 4 from hospitalization, for one month. At 3 and 6 months after discharge, follow-up checks, including abdominal ultrasound, complete blood count, liver and renal tests, were performed.

\section{Results}

Clinical and laboratory findings. Examination of the otherwise healthy young male patient with jaundice for 20 days and pruritus for 6 months following cholecystectomy revealed potential ephedra-induced liver injury with iron overload. In contrast to the obvious cholestasis, the liver injury was limited compared with other reported cases $(9,12)$, where patients were also self-treated with the same medication. CT scans indicated no focal lesions in the liver. Magnetic resonance cholangiopancreatography revealed a slightly dilatated post-cholecystectomy common bile duct with no evidence of choledocholithiasis (Fig. 1), moderate hepatosplenomegaly and intrahepatic lymphatic stasis. Furthermore, laboratory testing revealed hyperbilirubinemia (total/direct bilirubin, 253.8/134.9 $\mu \mathrm{mol} / \mathrm{l}$; normal range, 4.7-24/0-6.8 $\mu \mathrm{mol} / \mathrm{l}$ ), increased bile acid levels (338.6 $\mu \mathrm{mol} / 1$; normal range, 1.0-10.0 $\mu \mathrm{mol} / \mathrm{l}$ ), and normal prealbumin, alanine transaminase, aspartate aminotransferase, gamma-glutamyl transferase and alkaline phosphatase levels, prothrombin time and international normalized ratio value. The model for end-stage liver disease (MELD) score (13) was 17 with an R-factor value of 0.7 . Viral and autoimmune hepatitis were excluded. RUCAM scoring did not exclude DILI (6). Of note, the patient also suffered from chronic and mild hemolytic anemia with no identifiable cause (hemoglobin level, $109 \mathrm{~g} / \mathrm{l}$; normal range, 131-172 g/l) with an elevated mean corpuscular volume (91 fl), low haptoglobin level $(<0.1 \mathrm{~g} / \mathrm{l}$; normal range, 0.3-2.0 g/l) and moderate elevated percentages of reticulocytes $(2.5 \%$; normal range, $0.5-1.5 \%)$. Other findings included hyperferritinemia $(623 \mathrm{ng} / \mathrm{ml})$, increased transferrin saturation $(58.6 \%)$ and normal ceruloplasmin levels. RBC size variations may be readily observed under a microscope. During hospitalization, the RBC distribution width (15.3-17.8\%; normal range, 11.6-14.0\%) and the percentage of basophils (1.6-2\%; normal range; $<1.0 \%$ ) were consistently elevated. The levels of lactate dehydrogenase ( $\mathrm{LDH}$ ) were marginally increased, while those of ammonia were normal. These results indicated chronic mild to moderate macrocytic anemia. However, the Coombs tests (IgG and C3d), Isopropanol test, Ham's test, Glucose-6-phosphate dehydrogenase (G6PD) deficiency test and pyruvate kinase enzymatic activity were negative for autoimmune hemolytic anemia. Finally, high-performance liquid 
A

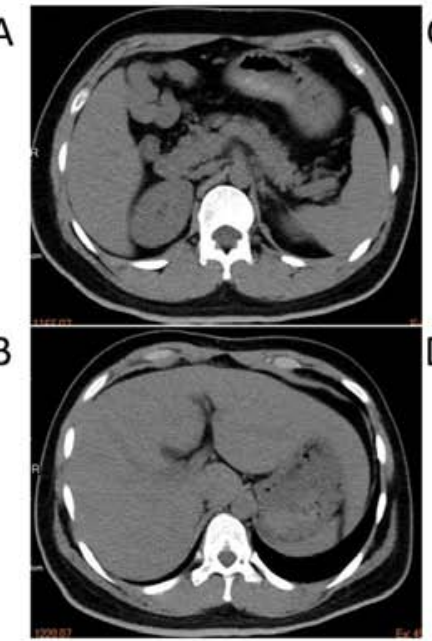

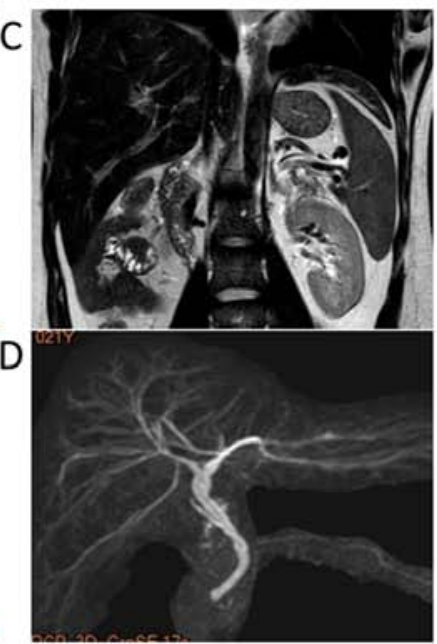

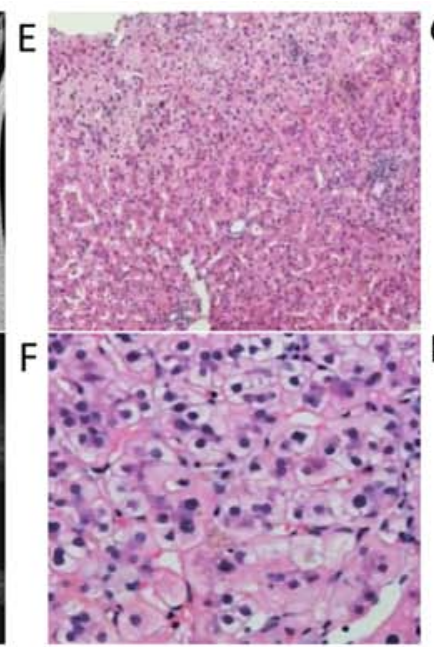

G CACCTGGTGGCACCG

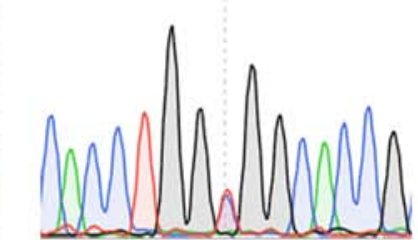

$H C A C C T G G C G G C A C B O$

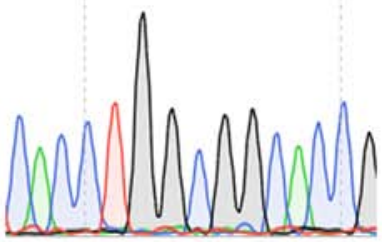

Figure 1. CT/MRCP and liver biopsy indicated characteristics of cholestasis with mild inflammatory events. CT scan revealed a slightly enlarged (A) spleen and (B) liver. (C) MRCP and (D) 3D reconstruction indicated slightly dilated intrahepatic bile ducts with no evidence of lithiasis (sequence parameters: T2-weighted; echo time, $120 \mathrm{msec}$; repetition time, 1,100 msec). (E) H\&E staining of the liver biopsy specimen revealing central lobular focal necrosis with mild portal inflammation (magnification, x100). (F) H\&E staining indicating cholestasis with dilated canaliculi, bile plug, apoptotic bodies and mild microvesicular steatosis (magnification, $\mathrm{x} 400$ ). (G) The $\mathrm{C}$ to T nucleotide substitution in the CDAN1 gene, causing R1067H, was verified by Sanger sequencing. (H) Sanger sequencing of the wild-type CDAN1 gene from the mother. CT, computed tomography; MRCP, magnetic resonance cholangiopancreatography; H\&E, hematoxylin and eosin; CDAN1, codanin 1.

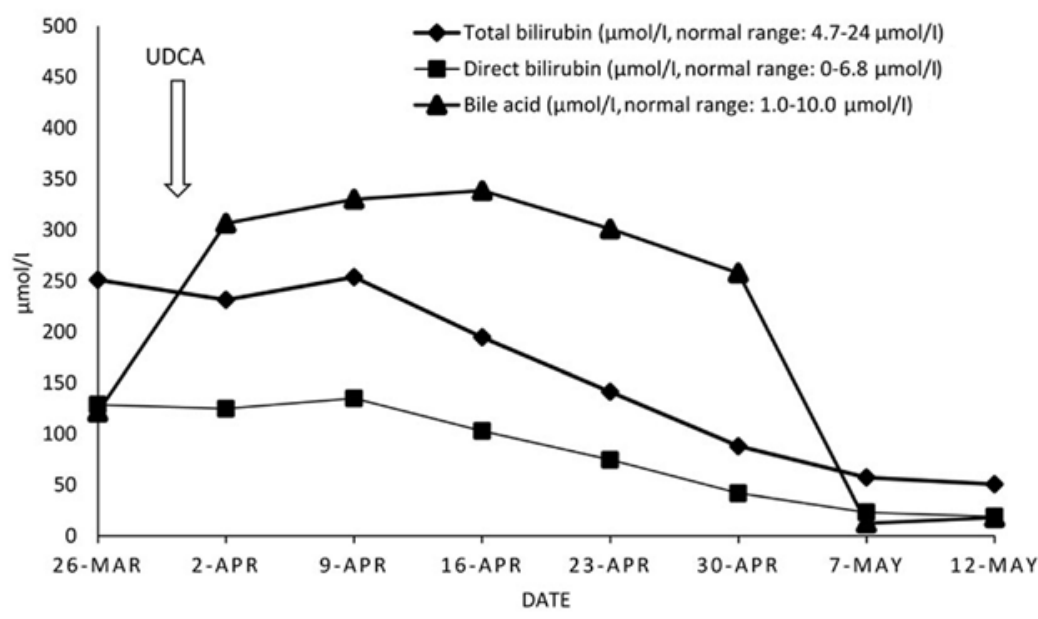

Figure 2. Dynamic changes in bilirubin and bile acid levels at various time-points during hospitalization. The arrow indicates the date of anti-cholestasis treatment. UDCA, ursodeoxycholic acid.

chromatography indicated normal levels of hemoglobin $\alpha$ ( $\mathrm{HbA}), \mathrm{HbA} 2$ and fetal hemoglobin, whilst no visible inclusion body was observed inside RBCs.

Liver biopsy and pathology. Staining of liver specimens with $\mathrm{H} \& \mathrm{E}$ revealed that the lobular structure remained intact, while intrahepatocyte cholestasis and bile plug were observed in the dilated canaliculus. In addition, a small number of foci of intralobular necrosis, apoptotic bodies, microvesicular steatosis and minimal portal inflammation were observed. The Masson trichrome staining was normal. The liver tissues were negative for hepatitis B surface and core antigens and for copper. Staining for cytokeratin 19 revealed slight ductular proliferation. The pathological data suggested that the patient suffered from acute cholestatic hepatitis, most likely caused by DILI (Fig. 1).
Disease-causing variation screening results. In addition to common homozygous polymorphisms in cholestasis-associated genes, including ATP binding cassette subfamily B member 11 (ABCB11) and tight junction protein 2 (TJP2), the pedigree genetic study revealed compound heterozygous variations in the congenital anemia-causing gene codanin 1 (CDAN1), including a novel rare mutation of paternal origin, namely p.R1067H (ClinVar accession no. SCV001448675). Apart from anemia, defects in the CDAN1 gene have also been associated with jaundice, iron overload, splenomegaly (14) and gallstones (Table I) (15). All of the aforementioned abnormalities were present in the patient of the present study.

Treatment and outcome. The patient was diagnosed with drug-induced bland cholestasis, with iron overload in the context of a rare form of congenital anemia. Since anemia 
Table I. Homozygous gene polymorphisms associated with cholestasis and congenital anemia causing mutations.

A, Common cholestasis-associated polymorphisms

\begin{tabular}{|c|c|c|c|c|c|c|}
\hline \multirow[b]{2}{*}{ Gene } & \multirow[b]{2}{*}{ AA Change } & \multicolumn{2}{|c|}{ Minor allele frequency } & \multirow[b]{2}{*}{ Disease (ID: OMIM or HGMD) } & \multirow{2}{*}{$\begin{array}{c}\text { Inheritance } \\
\text { pattern }\end{array}$} & \multirow[b]{2}{*}{ Note } \\
\hline & & GnomAD & 1000g_Chinese & & & \\
\hline$A B C B 11$ & p.V444A & 0.568838 & 0.664452 & $\begin{array}{l}\text { Cholestasis, benign recurrent } \\
\text { intrahepatic, } 2 \text { (605479); } \\
\text { cholestasis, drug-induced, } \\
\text { association with (CM071525) }\end{array}$ & $\begin{array}{l}\text { Autosomal } \\
\text { recessive }\end{array}$ & $\begin{array}{c}\text { Co-existed with another } \\
\text { heterozygous } \\
\text { cholestasis-related } \\
\text { polymorphism, } \\
\text { p.A1028A }\end{array}$ \\
\hline$T J P 2$ & $\begin{array}{l}\text { p.T909T } \\
\text { p.A913A }\end{array}$ & $\begin{array}{l}0.265188 \\
0.264951\end{array}$ & $\begin{array}{l}0.460133 \\
0.458472\end{array}$ & $\begin{array}{l}\text { Cholestasis, progressive familial } \\
\text { intrahepatic } 4(615878) \text {; } \\
\text { hypercholanemia, familial } \\
(607748)\end{array}$ & $\begin{array}{l}\text { Autosomal } \\
\text { recessive }\end{array}$ & - \\
\hline
\end{tabular}

B, Congenital anemia-associated mutations

\begin{tabular}{|c|c|c|c|c|c|c|}
\hline \multirow[b]{2}{*}{ Gene } & \multirow[b]{2}{*}{ AA Change } & \multicolumn{2}{|c|}{ Minor allele frequency } & \multirow[b]{2}{*}{ Disease (ID: OMIM or HGMD) } & \multirow{2}{*}{$\begin{array}{c}\text { Inheritance } \\
\text { pattern }\end{array}$} & \multirow[b]{2}{*}{ Note } \\
\hline & & GnomAD & 1000g_Chinese & & & \\
\hline CDAN1 & p.R1067H & l & Novel & $\begin{array}{l}\text { Dyserythropoietic anemia, } \\
\text { congenital, type Ia (224120) }\end{array}$ & $\begin{array}{l}\text { Autosomal } \\
\text { recessive }\end{array}$ & $\begin{array}{c}\text { Paternal origin } \\
\text { co-existed with another } \\
\text { two maternal } \\
\text { heterozygous variations } \\
\text { with unknown } \\
\text { significance }^{\mathrm{a}}\end{array}$ \\
\hline
\end{tabular}

${ }^{a}$ Two maternal variations with unknown significance included rs56046122 (intronic) and rs28661826 (exonic, also at exon 24). OMIM, Online Mendelian Inheritance in Man; HGMD, Human Gene Mutation Database; AA, amino acid.

was mild, the patient was treated only with UDCA, and cholestasis and pruritus swiftly subsided 2 weeks later (Fig. 2). Three and six months after discharge, follow-up examinations revealed that the patient remained in a good overall condition.

\section{Discussion}

The present study reported on the case of an otherwise healthy young male patient with an episode of cholestasis. According to the LiverTox.nih.gov website (16) (https://www.ncbi.nlm. nih.gov/books/NBK548711/), ephedra is known to promote liver injury. However, all reported cases, including hepatocellular and biliary injury (9), with or without co-treatment with steroids (12), were more severe compared to the present case. A comparison of the present and previously reported cases is provided in Table II. The previously reported cases were young adults, which is consistent with the current case. Furthermore, not only was the liver injury much milder in the present case, but a large proportion of the young male patients' liver functions were also well preserved, despite the fact that accumulated bile salts should have aggravated the hepatobiliary injury due to their inherent detergent activities (16). All of the above evidence indicated the presence of an intrahepatic protective molecule shielding the liver from further injury.

Of note, the clinical examination suggested a rare and mild type of chronic hemolytic anemia. These rare disorders may be caused by any genetic defect affecting RBCs, including Diamond-Blackfan anemia, congenital dyserythropoietic anemias, thalassemia, sickle cell disease, enzyme deficiency and red cell membrane disorders. These disorders are characterized by a variable degree of anemia, chronic extravascular hemolysis, reduced erythrocyte lifespan, splenomegaly, jaundice, biliary lithiasis and iron overload (17). Each type may require contradictory therapeutic approaches (3); therefore, the precise genetic diagnosis is of great importance. In the current case, a rare genetic variant was identified in the CDAN1 gene. This mutation is involved in the onset of rare congenital dyserythropoietic anemia type I (CDA-I), which is caused by bi-allelic mutations in the CDAN1 gene, usually accompanied by iron overload. In CDA-I, liver tests are commonly normal unless severe iron overload is present. However, unconjugated bilirubin and LDH levels are usually elevated due to hemolysis and ineffective erythropoiesis. It has been reported that abdominal ultrasound in patients with CDA-I reveals universal splenomegaly (14), while $50-60 \%$ of adult patients have gallstones (15). In the current case, all the aforementioned clinical 
Table II. Comparison among ephedra-administered cases.

\begin{tabular}{lccc}
\hline Item & Case by Nadir et al $(9)$ & Case by Singh et al (12) & Present case \\
\hline Age (years) & 33 & 24 & 21 \\
Sex & Female & Male & Ephedra \\
Medication & Ephedra & Ephedra with steroids & Bland cholestatic \\
Pattern & Hepatocellular & Bland cholestatic & 37 \\
ALT $(\mathrm{U} / \mathrm{l})$ & 1586 & 237 & 104 \\
ALP $(\mathrm{U} / \mathrm{l})$ & 175 & 129 & 253.8 \\
Bilirubin $(\mu$ mol/l) & 136.80 & 906.3 & 12 \\
Prothrombin time (sec) & 13.2 & $\mathrm{NA}$ & Yes \\
Jaundice & Yes & Yes & 1 \\
Recovery time (months) & 4 & 3 &
\end{tabular}

Normal range for ALT (10-64 IU/l), ALP (38-126 IU/l), Bilirubin (4.7-24 $\mu \mathrm{mol} / \mathrm{l})$, Prothrombin time (10-14 sec). ALT, alanine aminotransferase; ALP, alkaline phosphatase; NA, not available.

features were present and the patient had splenomegaly and a history of gallstones. Furthermore, the dysregulation of erythropoiesis was evidenced by the marginally elevated percentage of reticulocytes relative to the degree of anemia, and constantly by the increased number of basophils in the peripheral blood of the patient. In this case, genetic testing is recommended, since it may significantly reduce the time required for accurate molecular diagnosis and prevent invasive bone marrow aspiration. To the best of our knowledge, the novel p.R1067H mutation identified in the present study, located at the disease-related hotspot exon 24 (18), has not been previously reported and is adjacent to the well-established disease-related p.D1043V mutation. Computational prediction tools, including SIFT (sift.jcvi.org), PolyPhen-2 (genetics. bwh.harvard.edu/pp2), mutation taster (www.mutationtaster. org), PROVEAN (provean.jcvi.org/index.php), SNPs \& GO (snps-and-go.biocomp.unibo.it), PANTHER (pantherdb.org), PhD-SNP (snps.biofold.org/phd-snp/phd-snp.html), I-Mutant (folding.biofold.org/cgi-bin/i-mutant2.0.cgi) and SNAP (www. rostlab.org/services/SNAP), unanimously support the deleterious effect of mutations of this gene (19-21).

While the hemolytic process may predispose the patient's liver to cholestatic injury, most likely through iron overload, lithiasis and splenomegaly, it may also guarantee a steady flow of slightly elevated bilirubin in the blood (hyperbilirubinemia). Bilirubin, a pigment with potent anti-oxidative properties (22), is known to protect against inflammation-mediated diseases $(23,24)$ all-cause mortality $(25)$ and even possesses therapeutic value $(23,24,26)$. However, the intrahepatic protective role of mildly-elevated unconjugated bilirubin has not been reported in the literature, to the best of our knowledge. Therefore, the present case provided hint that the hemolysis-mediated mild hyperbilirubinemia may prepare and protect the liver from further injury.

In conclusion, the present study reported on a case of DILI, in the context of a mild form of rare congenital anemia resulting in bland cholestasis. Hemolysis-induced mildly-elevated unconjugated bilirubin may contribute to the milder DILI observed in the current case compared to the previously reported cases. Therefore, the present study highlighted the importance of genetic pedigree analysis, particularly when a given condition is manifested in additional family members. In addition, a novel rare mutation in the CDAN1 gene was identified in the present case, enhancing the spectrum of mutations known to be associated with such pathologies.

\section{Acknowledgements}

Not applicable.

\section{Funding}

The present study was supported by grants from the National Natural Science Foundation of China (grant no. 82002126), the Shanghai Jiao Tong University (SJTU) medical X engineering project (grant no. YG2017MS63) and the Excellent Youth B Project (grant no. GCQN-2017-B20).

\section{Availability of data and materials}

The next-generation sequencing datasets generated and analyzed during the current study are available in the National Center for Bioinformatics Information (NCBI) Sequence Read Archive repository (accession no. PRJNA690696; https://www. ncbi.nlm.nih.gov/sra/PRJNA690696).

\section{Authors' contributions}

YH drafted the manuscript and performed the laboratory tests. YZ, WT, LC and YC performed the follow-up checks and analyzed the clinical data. The experiments were designed by QG and XZ. QG and XZ checked and approved the authenticity of the raw data. All authors read and approved the final manuscript.

\section{Ethics approval and consent to participate}

The present study was approved by the Ethics Committee of Ruijin Hospital (Shanghai, China; ID: 2016-17). Written informed consent was provided by the patient. 


\section{Patient consent for publication}

The patient and his parents provided written informed consent for publication of the results.

\section{Competing interests}

The authors declare that they have no competing interests.

\section{References}

1. Gambale A, Iolascon A, Andolfo I and Russo R: Diagnosis and management of congenital dyserythropoietic anemias. Expert Rev Hematol 9: 283-296, 2016.

2. Russo R, Andolfo I, Manna F, Gambale A, Marra R, Rosato BE, Caforio P, Pinto V, Pignataro P, Radhakrishnan $\mathrm{K}$, et al: Multi-gene panel testing improves diagnosis and management of patients with hereditary anemias. Am J Hematol 93: 672-682, 2018.

3. Bolton-Maggs PH, Stevens RF, Dodd NJ, Lamont G, Tittensor P and King MJ; General Haematology Task Force of the British Committee for Standards in Haematology: Guidelines for the diagnosis and management of hereditary spherocytosis. $\mathrm{Br}$ J Haematol 126: 455-474, 2004.

4. European Association for the Study of the Liver. Electronic address: easloffice@easloffice.eu; Clinical Practice Guideline Panel: Chair; Panel members and EASL Governing Board representative: EASL clinical practice guidelines: Drug-induced liver injury. J Hepatol 70: 1222-1261, 2019.

5. Lucena MI, Sanabria J, García-Cortes M, Stephens C and Andrade RJ: Drug-induced liver injury in older people. Lancet Gastroenterol Hepatol 5: 862-874, 2020.

6. Danan $\mathrm{G}$ and Teschke R: RUCAM in drug and herb induced liver injury: The update. Int J Mol Sci 14: 17, 2015.

7. Russmann S, Jetter A and Kullak-Ublick GA: Pharmacogenetics of drug-induced liver injury. Hepatology 52: 748-761, 2010.

8. Garcia-Cortes M, Robles-Diaz M, Stephens C, Ortega-Alonso A, Lucena MI and Andrade RJ: Drug induced liver injury: An update. Arch Toxicol 94: 3381-3407, 2020.

9. Nadir A, Agrawal S, King PD and Marshall JB: Acute hepatitis associated with the use of a Chinese herbal product, ma-huang. Am J Gastroenterol 91: 1436-1438, 1996.

10. 1000 Genomes Project Consortium; Auton A, Brooks LD, Durbin RM, Garrison EP, Kang HM, Korbel JO, Marchini JL, McCarthy S, McVean GA and Abecasis GR: A global reference for human genetic variation. Nature 526: 68-74, 2015.

11. Iezzoni JC: Diagnostic histochemistry in hepatic pathology. Semin Diagn Pathol 35: 381-389, 2018.

12. Singh C, Bishop P and Willson R: Extreme hyperbilirubinemia associated with the use of anabolic steroids, health/nutritional supplements, and ethanol: Response to ursodeoxycholic acid treatment. Am J Gastroenterol 91: 783-785, 1996.
13. Brown RS Jr, Kumar KS, Russo MW, Kinkhabwala M, Rudow DL, Harren P, Lobritto S and Emond JC: Model for end-stage liver disease and Child-Turcotte-Pugh score as predictors of pretransplantation disease severity, posttransplantation outcome, and resource utilization in United Network for Organ Sharing status 2A patients. Liver Transpl 8: 278-284, 2002.

14. Heimpel H, Schwarz K, Ebnöther M, Goede JS, Heydrich D, Kamp T, Plaumann L, Rath B, Roessler J, Schildknecht O, et al: Congenital dyserythropoietic anemia type I (CDA I): Molecular genetics, clinical appearance, and prognosis based on long-term observation. Blood 107: 334-340, 2006.

15. Shalev H, Kapleushnik Y, Haeskelzon L, Degani O, Kransnov T, Sphilberg O, Moser A, Yaniv I and Tamary H: Clinical and laboratory manifestations of congenital dyserythropoietic anemia type I in young adults. Eur J Haematol 68: 170-174, 2002.

16. LiverTox: Clinical and Research Information on Drug-Induced Liver Injury. [Internet]. National Institute of Diabetes and Digestive and Kidney Diseases, Bethesda (MD), 2012.

17. Zaninoni A, Fermo E, Vercellati C, Marcello AP, Barcellini W and Bianchi P: Congenital hemolytic anemias: Is there a role for the immune system? Front Immunol 11: 1309, 2020.

18. Roy NBA and Babbs C: The pathogenesis, diagnosis and management of congenital dyserythropoietic anaemia type I. Br J Haematol 185: 436-449, 2019.

19. Borgio JF, Al-Madan MS and AbdulAzeez S: Mutation near the binding interfaces at alpha-hemoglobin stabilizing protein is highly pathogenic. Am J Transl Res 8: 4224-4232, 2016.

20. AbdulAzeez S and Borgio JF: In-silico computing of the most deleterious nsSNPs in HBA1 Gene. PLoS One 11: e0147702, 2016.

21. Abdulazeez S, Sultana S, Almandil NB, Almohazey D, Bency BJ and Borgio JF: The rs61742690 (S783N) single nucleotide polymorphism is a suitable target for disrupting BCL11A-mediated foetal-to-adult globin switching. PLoS One 14: e0212492, 2019.

22. Fujiwara R, Haag M, Schaeffeler E, Nies AT, Zanger UM and Schwab M: Systemic regulation of bilirubin homeostasis: Potential benefits of hyperbilirubinemia. Hepatology 67: 1609-1619, 2018.

23. Vitek L, Bellarosa C and Tiribelli C: Induction of mild hyperbilirubinemia: Hype or real therapeutic opportunity? Clin Pharmacol Ther 106: 568-575, 2019.

24. Boon AC, Hawkins CL, Coombes JS, Wagner KH and Bulmer AC: Bilirubin scavenges chloramines and inhibits myeloperoxidase-induced protein/lipid oxidation in physiologically relevant hyperbilirubinemic serum. Free Radic Biol Med 86: 259-268, 2015.

25. Bulmer AC, Bakrania B, Du Toit EF, Boon AC, Clark PJ, Powell LW, Wagner KH and Headrick JP: Bilirubin acts as a multipotent guardian of cardiovascular integrity: More than just a radical idea. Am J Physiol Heart Circ Physiol 315: H429-H447, 2018.

26. Vitek $\mathrm{L}$ and Tiribelli C: Bilirubin, intestinal integrity, the microbiome, and inflammation. N Engl J Med 383: 684-686, 2020. 